\title{
Cognitive Radio RF: Overview and Challenges
}

\author{
Van Tam Nguyen, ${ }^{1}$ Frederic Villain, ${ }^{2}$ and Yann Le Guillou ${ }^{3}$ \\ ${ }^{1}$ Institut Mines-Telecom, Telecom ParisTech, LTCI CNRS UMR 5141, 75634 Paris, France \\ ${ }^{2}$ BL TV Front End, NXP, 14460 Colombelles, France \\ ${ }^{3}$ Renesas Mobile Corporation, 35517 Cesson Sevigne Cedex, France \\ Correspondence should be addressed to Van Tam Nguyen, vtnguyen@telecom-paristech.fr
}

Received 4 November 2011; Revised 6 February 2012; Accepted 7 February 2012

Academic Editor: Christophe Moy

Copyright ( $\odot 2012$ Van Tam Nguyen et al. This is an open access article distributed under the Creative Commons Attribution License, which permits unrestricted use, distribution, and reproduction in any medium, provided the original work is properly cited.

Cognitive radio system (CRS) is a radio system which is aware of its operational and geographical environment, established policies, and its internal state. It is able to dynamically and autonomously adapt its operational parameters and protocols and to learn from its previous experience. Based on software-defined radio (SDR), CRS provides additional flexibility and offers improved efficiency to overall spectrum use. CRS is a disruptive technology targeting very high spectral efficiency. This paper presents an overview and challenges of CRS with focus on radio frequency (RF) section. We summarize the status of the related regulation and standardization activities which are very important for the success of any emerging technology. We point out some key research challenges, especially implementation challenges of cognitive radio (CR). A particular focus is on RF front-end, transceiver, and analog-to-digital and digital-to-analog interfaces which are still a key bottleneck in CRS development.

\section{Introduction}

The information and communication technology industry is today faced with a global challenge: develop new services with improved quality of service (QoS) and at the same time reduce its environmental impact. Clearly, there is a deep need of global efficiency not only in the energy domain, but also in the spectral domain.

Indeed, the gap between wireless supply and demands widens. It is feared that an imminent spectrum crisis, in which exploding demand from smart phones will soon overwhelm the wireless capacity, will occur. The problem is the lack of new spectrum available to wireless data carriers. Smartphone data traffic is growing so fast that if nothing is done they will use up the available spectrum. This just affects not only smart phones but also all wireless devices. A huge amount of spectrum is required for broadband use in the future as suggested in National Broadband Plan (NBP) in USA and the digital agenda in Europe.

In order to address the problem of spectrum usage efficiency, the cognitive radio (CR) concept was proposed
$[1,2]$. The detailed definition of cognitive radio systems (CRSs) will be given in Section 2. Cognitive radio technology has the potential of being a disruptive force within spectrum management.

A very popular example is opportunistic radio or opportunistic spectrum access whose principle is temporal, spatial, and geographic "reuse" of licensed spectrum as shown in Figure 1 where an "unlicensed" secondary user (SU) can be permitted to use licensed spectrum, provided that it does not interfere with any primary users (PUs). In that way, the efficiency of spectrum usage is significantly improved. Various measurements of spectrum utilization have shown that spectrum is underutilized, in the sense that the typical duty cycle of spectrum usage at a fixed frequency and at a random geographical location is low. This means that there are many "holes" in the radio spectrum that could be exploited [3]. Opportunistic radio system should be able to exploit these spectrum holes by detecting them and using them in an opportunistic manner. Because of the outstanding propagation characteristic in the television (TV) bands with strong wall and floor penetration capability, long 


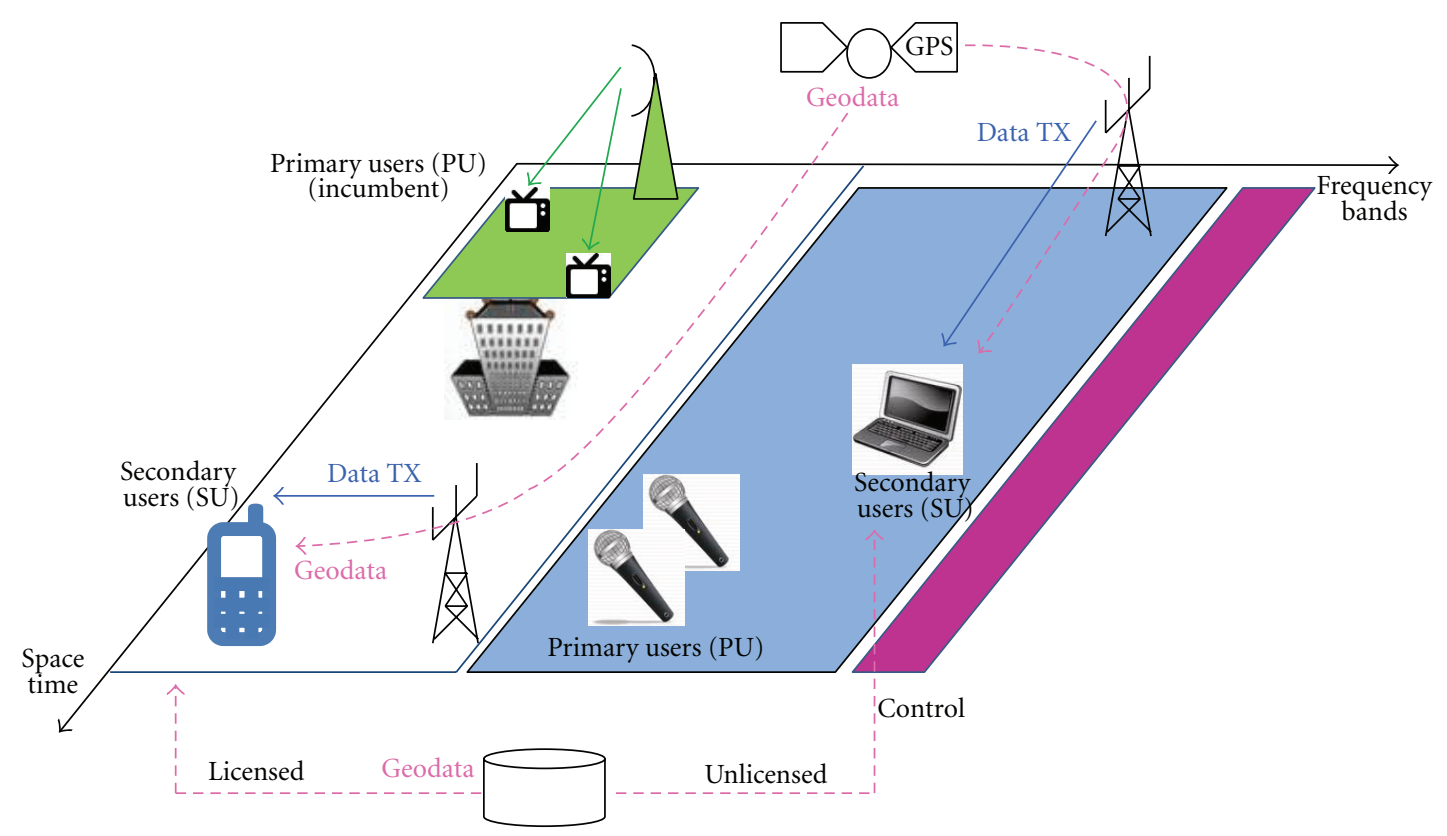

FIgURE 1: Opportunistic spectrum access overview.

range, and flexible bandwidth, it could be used to allow a brand-new class of services and increase the limited capacity of existing systems.

But CRS is not only limited to opportunistic spectrum access. Also, it includes heterogeneous networks where a heterogeneous radio framework management is performed. Current spectrum allocation approaches, fixed by nature, do not allow for the allocation of frequency bands to different radio access technologies (RATs) dynamically. However, the coexistence and cooperation of diverse technologies, which form part of a heterogeneous infrastructure, have brought about the possibility of flexibly managing the spectrum in a dynamic manner. No longer are fixed frequency bands guaranteed to apply to specific RATs, but conversely, through intelligent management mechanisms, bands can be allocated to RATs dynamically in a way such that the capacity of each RAT is maximized and interference is minimized. on long terms, will be considered also the flexibility in spectrum management where network operator may employ different RATs dynamically over time/frequency/location and acquire or exchange the spectrum usage rights. The devices may autonomously and dynamically adapt to the diverse heterogeneous radio networks [4].

This new technology opens up many potential applications and exciting opportunities [5]. For example, the rural connectivity, content distribution networks, city and campus wide coverage, and giant wireless hotspots could benefit from the new spectrum access and management.

This paper is organized as follows: after this introduction, the definition and high level concept of CRS is presented in Section 2. In Section 3, the status on regulation and standardization activities are described. Section 4 will present the research challenged related to CRS. Section 5 will describe the implementation challenges of CR devices. The conclusion of the paper is drawn in Section 6.

\section{Definition and High Level Concept}

There are different definitions of CRS, from many authors and organizations. The definition giving the common understanding about CRS and now adopted for most is from International Telecommunication Union (ITU) [6]. CRS is a radio system employing technology that allows the system:

(i) to obtain knowledge of its operational and geographical environment, established policies, and its internal state (cognitive capability);

(ii) to dynamically and autonomously adjust its operational parameters and protocols according to its obtained knowledge in order to achieve predefined objectives (reconfigurable capability);

(iii) to learn from the results obtained (learning capability).

At high level concept presented in Figure 2, the main components of the CRS are the intelligent management system and reconfigurable radios $[7,8]$. CRS is also able to take action including obtaining knowledge, making decision, reconfiguration, and learning. The knowledge used by the CRS includes knowledge about operational radio and geographical environment, internal state, established policies, usage patterns, and users' needs.

The methods to obtain this knowledge include getting information from component of the CRS, spectrum sensing, access to the cognitive pilot channel (CPC), geolocation, and database access. Using the obtained knowledge, the CRS 


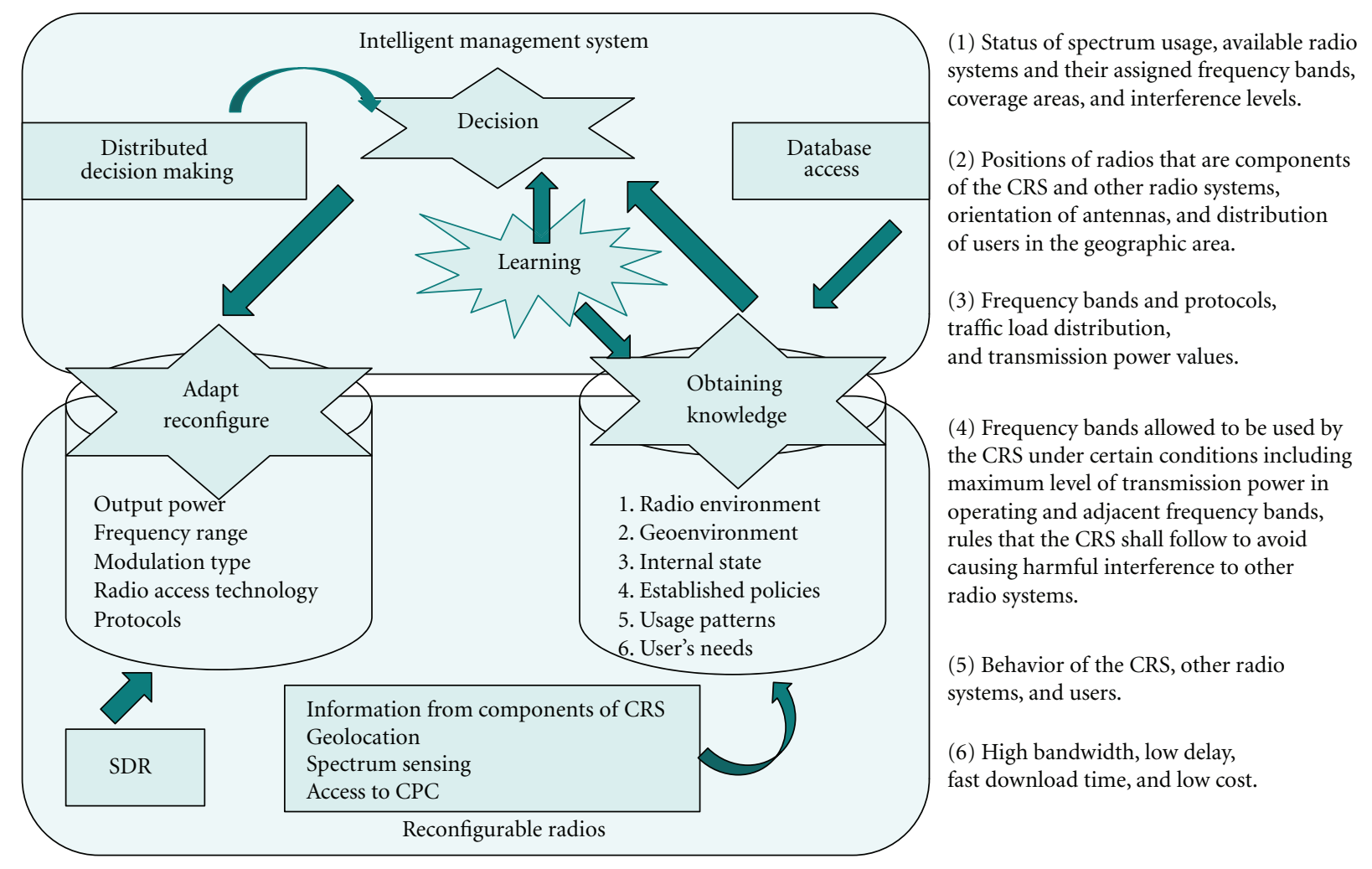

FIGURE 2: High-level CRS concept.

dynamically and autonomously makes reconfiguration decisions according to some predefined objectives, for example, in order to improve efficiency of spectrum usage. Based on the decisions made, the CRS adjusts operational parameters and protocols of its reconfigurable radios. Such parameters include output power, frequency range, modulation type, and radio access technology (RAT) protocols.

Software-defined radio (SDR) approach is used to implement the reconfigurations. Also, the CRS can learn from its decisions to improve its future decisions. The results of learning contribute to both obtaining knowledge and decision making.

CRS can be classified into two types: heterogeneous CRS and spectrum sharing CRS. The first type uses the network centric approach where one or several operators operate several radio access networks (RANs) using the same or different RATs. Frequency bands allocated to these RANs are fixed. Cognitive network optimizes radio resources and improves the QoS. The second type of CRS is sharing CRS, where several RANs using the same or different RATs can share the same frequency band by using the unoccupied subbands in an intelligent and coordinated way. Most of standardization activities are related to this type of CRS.

\section{Status in Regulations and Standardizations}

3.1. Regulations. The major regulatory agencies are developing rules for the unlicensed use of TVWS such as the FCC in the United States, Ofcom in the UK, and the Electronic Communications Committee (ECC) of CEPT in Europe.

The FCC provided the final rules for TVWS in 2010 [9]. There is ongoing proceeding for secondary use of the $2.36 \mathrm{GHz}$ to $2.4 \mathrm{GHz}$ band for medical area networks. Other opportunistic spectrum access beyond the already completed TVWS proceedings and cognitive techniques to better utilize the radio spectrum are currently under investigation.

Ofcom has also made significant progress in developing regulations for the TVWS with a first public consultation in 2009 [10]. The statement on white spaces devices and implementation of geolocation databases was released on September 1st, 2011 [11]. The detailed rules will be released in the future.

The ECC studied the technical and operational requirements for the operation of CRS in the WS of the UHF broadcasting band $(470-790 \mathrm{MHz})$ [12]. This work is used as the starting point for regulatory activities within the ECC.

3.2. Standardizations. Currently international standardization of CRS is performed at all levels (ITU, IEEE, ETSI, and ECMA) $[7,13]$. They are considering multiple deployment scenarios and business directions.

In ITU, Working Party (WP) $1 \mathrm{~B}$ has worked on the definition of SDR and CRS and their relationship and summarized the technical and operational studies, and relevant recommendations. It has considered the SDR and CRS usage scenarios in different radio services and regulation 
implications. The WP 5A is currently addressing the definition, description, and application of CRS in the land mobile service.

IEEE is very active in CRS. In 802 WGs (LAN/MAN), the activity to define CRSs is currently performed in the 802.11 and 802.22 , while the activity to specify components of a CRS is currently performed in $802.19,802.21$, and 802.22. $802.11 y$ is an amendment for $3650-3700 \mathrm{MHz}$ operation in USA defining new regulatory classes, transmit power control, and dynamic frequency selection for 802.11 to share frequency bands with other users. Draft standard P802.11af is an amendment for TVWS operation defining standardized modifications to both the 802.11 physical (PHY) layers and medium access control (MAC) sublayer to meet the legal requirements for channel access and coexistence in the TVWS. Draft standard P802.19.1 concerns TVWS coexistence methods. IEEE 802.21 focuses on media independent handover services enabling the optimization of handover between heterogeneous IEEE 802 networks, and facilitating handover between IEEE 802 networks and cellular networks. The draft standard P802.22 is on policies and procedures for operation in the TV bands. It specifies the air interface, including the cognitive MAC and PHY, of pointto-multipoint wireless regional area networks, operating in the unlicensed TV bands between $54 \mathrm{MHz}$ and $862 \mathrm{MHz}$. Draft standard P802.22.1 is to enhance harmful interference protection for PUs operating in TV bands.

The IEEE DySPAN standards committee, whose predecessor is the IEEE P1900 standards committee, develops IEEE standards for radio and spectrum management with focus on improved use of spectrum. Its working groups and resulting standards numbered in the 1900 range propose new techniques and methods of dynamic spectrum access (DSA), which requires managing interference and coordination of wireless technologies and includes network management and information sharing. The IEEE 1900.1 standard related to terminology and concepts was published in 2008. The IEEE 1900.4 published in 2009 , defines management system to support network-terminal distributed optimization of radio resource usage and improvement in QoS in heterogeneous wireless networks. The IEEE 1900.4a working group is defining architecture and interfaces for DSA networks in TVWS. The IEEE 1900.5 standard is dedicated exclusively to policy language requirement and architecture. The IEEE 1900.6 standard deals with spectrum sensing interfaces and data structures. IEEE P1900.7 is a draft standard on radio interface for white space dynamic spectrum access radio systems supporting fixed and mobile operation. It specifies a radio interface including MAC sublayer and PHY layer of white space dynamic spectrum access radio systems supporting fixed and mobile operation in white space frequency bands, while avoiding causing harmful interference to incumbent users in these frequency bands. The standard provides means to support P1900.4a for white space management and P1900.6 to obtain and exchange sensing-related information (spectrum sensing and geolocation information). Recently the ad hoc on dynamic spectrum access in vehicular environments was created. The purpose is to consider interest in,
TABLE 1: Summary of CRS activities in standardization and open issues.

\begin{tabular}{lc}
\hline Functionality & Covering organization \\
\hline Definitions & IEEE SCC 41, ETSI, ITU-R \\
Coexistence & IEEE 802.19, IEEE SCC 41 \\
SDR & IEEE SCC 41, SDR Forum, ITU-R, OMG, ETSI \\
Radio interfaces & IEEE 802.22, 3GPP, ECMA \\
Heterogeneous & ETSI, IEEE SCC 41 \\
access & \\
Spectrum & - \\
sensing & - \\
Testing & IEEE 802.22, IEEE SCC 41 \\
Networking & - \\
Security & \\
\hline
\end{tabular}

feasibility of, and necessity of developing a standard radio interface for vehicular communications.

The ETSI Reconfigurable Radio Systems (RRS) Technical Committee (TC) is also active in standardizing SDR and CRS [14]. TC RRS main responsibility is to carry out standardization activities related to reconfigurable radio systems (RRS) encompassing both SDR and CR with a focus on specific needs of the European Regulatory Framework, and CR/SDR TV white space standards adapted to the digital TV signal characteristics in Europe. Two out of the four working groups within ETSI RRS have activities resulting in standardization of potential regulatory aspects of CRS and SDR. Working group 3 has proposed and investigated the feasibility of standardizing a functional architecture for management and control of reconfigurable radio systems and cognitive pilot channel. SDR-related standardization is considered for both base station and mobile device. Working group 2 relies mainly on mobile device SDR related interface standardization. ETSI RRS is also working on operation in WS frequency bands and coexistence architecture for cognitive radio and investigating security and threats issues.

ECMA-392 released in 2009, specifies MAC and PHY for personal/portable cognitive wireless networks operating in TVWS, a MUX sublayer for higher layer protocols and a number of incumbent protection mechanisms.

3GPP is also interested in standardizing CR-like features in its future releases. For example, the idea of a cognitive reference signal is proposed through which each RAN can broadcast the interference level, frequency bands, and RATs of other networks, and other information that can help newly joined user equipment to choose the best RAN.

We summarize different aspects of standardization for CRS in Table 1. There are a lot of standardization activities for CRS with a lot of overlapping. However, many open issues have not been covered at all until now, especially the security issues.

\section{Research Challenges}

CRS covers multidisciplinary areas attracting a large number of research works with many interesting obtained results. 
The challenges remain numerous, namely, intelligence distribution and implementation, delay/protocol overhead, cross-layer design, security, sensing algorithms, and flexible hardware design. Due to the huge amount of published papers and the interdisciplinary nature of the topic, it is very difficult to provide an exhaustive analysis of all research works available on CRS. The purpose of this section is therefore to briefly describe challenges which are yet open and current under debate in the framework of research on CRS.

4.1. Decision Making. As CRS is driven by a decision making, the first relevant research challenge is where and how the decision (e.g., the decision on spectrum availability, strategy for selecting channel for sensing or access, or how to optimize radio performance) should be taken. The first issue is directly related to whether the cognitive process should be implemented in a centralized or distributed fashion. This aspect is more critical not only for cognitive networks, where intelligence is more likely to be distributed, but also for cognitive radios, as decision making could be influenced by collaboration between them and also with other devices. The second issue is the choice of the decision algorithms (e.g., neural networks, genetic algorithms, antcolony optimization, etc.) which should be customized to fulfill the CRS requirements.

4.2. Learning Process. Research in machine learning has grown dramatically recently, with significant amount of progress. One of the important aspects of the learning mechanisms is whether the learning performed is supervised or unsupervised. In the context of a CRS, either technique may be applied. The first challenge of learning is to avoid wrong choices before a feasible decision, especially in autonomous or unsupervised learning process. The second issue is to concretely define learning process in the context of CRS, its objectives and contributions.

In terms of implementation and algorithm design, the cognitive functionalities, which are related to enabling devices or networks to learn from past decisions to improve their behavior, are too much complex. The design of the learning algorithm represents by itself a challenge, and measurements which should be employed by learning open new issues related to which measurements to use and how to perform them.

4.3. Cross-Layers. While the aspect of interprotocol interaction is included in the concept of cognitive network as means to support user and applications requirement, no relevant and comprehensive analysis is available to address the performance and, in general, the behavior of applications and networks based on CRS technology. The design of cognitive or self-organized network is itself a challenging task, in particular, the outer and inner loops coordination, the networking middleware for knowledge exchange, and intersystem networking for sharing and cooperation.

Challenge is also in the design of high layers including MAC sublayer and network layer, spectrum management functions integrated at the different layers of the network protocol stack, cognitive radio resource management and coordination, various protocols and routings. Many technologies will be using multiple frequency bands. As a result, challenges in interoperability, including coexistence, cooperation and collaboration for devices, and networks signaling with cross-layer interfaces and interlayer signaling are to be solved.

Although many papers deal with the cross-layer design, the issues addressed are still specific and tailored to a given technology with the lack of its general. We need to provide cross-layer designs for more general classes of communications schemes.

4.4. Security. The challenges of employing CRS include that of ensuring secure devices operations. Security in this context includes enforcement of rules. Enforcement for static systems is already a challenge due to the amount of resources necessary to authorize equipment, the requirement of obtaining proof that violations have occurred, and the determination of the violator' identities. As the systems become more dynamic, there is an increase in the number of potential interaction that can lead to a violation. Additionally, this leads to a decrease of the time and special scales of these interactions. Both of these changes will amplify the enforcement challenges.

The first issue is on equipment authorization, especially on evaluation criteria and security certification. It becomes even more problematic with the employment of self-learning mechanisms. Software and hardware certification will not provide sufficient assurances that the device conforms to the operational envelopes.

Software certification and the security of the software are also challenging area, especially when software provides the control of dynamic systems. The security of that software is critical to ensure that rogue behavior is not programmed into the devices.

The number of combination of interactions is high and the mobility and the agility of CRS is great, so the monitoring mechanisms are challenging tasks.

Also, security is to deal at the protocol layer with key exchanges which are very adapted to a highly reliable physical layer and centralized network. However, in CRS with distributed cognitive networks, traditional cryptographic schemes are not adapted.

4.5. Sensing. Following challenge is about spectrum sensing, especially on the accuracy on spectrum occupancy decision, sensing time, and malicious adversary, taking into account the fundamental limits of spectrum sensing algorithms due to noise uncertainty multipath fading and shadowing [15]. In order to solve hidden PU problem and mitigate the impact of these issues, cooperative spectrum sensing has been shown to be an effective method to improve the detection performance by exploiting spatial diversity in the observations of spatially located CRs [16]. Challenges of cooperative sensing include reducing cooperation overhead, developing efficient information sharing algorithms. The 
coordination algorithm for cooperation should be robust to changes and failures in the network, and introduce a minimum amount of delay.

The most prominent hardware trial for spectrum sensing thus far has been the FCC field trial conducted in 2008 by the office of engineering and technology [17]. Although the spectrum sensing approach exhibited good sensitivities satisfying stringent regulation requirements, the future spectrum sensing hardware should improve the receiver selectivity and receiver desensitization, especially when the adjacent channels have high powers. The geolocation databasebased approach is able to identify occupied channels with $100 \%$ accuracy. However, for identification of unoccupied channels, it did not exhibit the best performance, presumably due to incomplete information in the database. This shows that the spectrum sensing alone works to some degree, but the performance could be further enhanced especially in the identification of occupied channels. Combining a geolocation database with spectrum sensing may be a better option provided that the CR device cost and power dissipation are decreased.

4.6. Geolocation. Geolocation is an important CR enabling technology due to the wide range of applications that may results from a radio being aware of its current location and possibility being aware of its planned path and destination. When CRS uses the geolocation technology for location determination combined with a database look-up, each access point (AP) may be connected to one, or multiple databases which provide information on the unused TVWS channels that are available at the location of AP and they provide also information on maximum transmit power levels usable in each channel. Furthermore, the use of master-slave technology is encouraged so that the necessary functionalities for database lookup and channel selection need to be implemented only in APs. It keeps the complexity and cost of end-user devices to a minimum.

However, the challenges in this area are who and how to implement the data base, how to feed it. Providing incumbent (or PUs) databases requires knowledge of the locations of CR devices whose precision should be specified. If global positioning service (GPS) is equipped and CR devices are outdoor, obtaining their geolocations may still be less a technical challenge. If no GPS is available or if CR devices are indoor, then obtaining geolocations becomes a challenging task.

\section{Implementation Challenges}

Although the theoretical research for CRS is blooming, with many interesting results, hardware implementation and system development are progressing at a slower pace, because of the complexities involved in designing and developing CRS [17]. In this section, we present the implementation challenges of CR in the system on chip (SoC) integration's perspective.

The first SDR architecture was proposed by Mitola and Maguire [1], in which the RF and analog processing are reduced to only a pair of data converters, thus providing the maximum flexibility and programmability through the digital processing block. This idealistic approach, however, suffers from the poor tolerance of to the interferers. In many wireless applications, a small desired signal could be accompanied by several large in-band signals created by nearby transmitters of the same communication standard or outof-band blockers caused by any transmitter. At times, these blockers could be as much as about $100 \mathrm{~dB}$ larger than the desirable received signal, which, due to the lack of any filtering in this idealistic approach would demand an impractical dynamic range of about $100 \mathrm{~dB}$ on the ADC. This long-term requirement is very far beyond the limits of the technology available as will be shown in ADC/DAC challenges subsection. Research on high-performances ADC/DAC is going on with significant progress, especially with hybrid-filter bank and time-interleaving architectures.

For short- and midterm implementation, the block diagram of a CR may be presented in Figure 6. In order to operate on very wide band or multibands simultaneously, parallel processing is employed from antennas to analog to digital interfaces as shown in Figure 3. Multiantennas are necessary for multi-input-multioutput (MIMO) operation and/or multibands operation. After antennas, a passive module is used for switching or duplexing, RF filtering, and impedance matching between antennas and power amplifiers (PAs). This module is composed of a range of submodules in order to cover a wide bandwidth or enable simultaneous communications. Then multireceiver ( $\mathrm{Rx})$ and multitransmitter (Tx) are followed before a multi-ADC/DACs module. A high performance and very flexible digital baseband carries out not only all conventional processing for modulation and demodulation, coding and encoding, and so forth, but also digital filtering, dc offset cancellation, digital automatic gain control, calibration and correction of analog errors and non linearities. Combining with control plane and sensor, a feedback from baseband to RF front-end and transceiver are necessary to boost the performance of the analog part. The challenges of RF front-end and transceiver in the short-/midterm are to reduce the off-chip and passive components, increase their frequency-agility, minimize the power dissipation, and reduce area. These challenges will be discussed more in detail in the next two subsections.

CR needs to adapt transmission and receiver parameters to avoid causing interference to PUs and maximize spectral efficiency. To avoid causing interference, numerous techniques can be used and combined such as frequency tuning (adaptive frequency hopping, dynamic frequency selection and RF band switching), OFDM subchannelization, channel aggregation, time multiplexing, power control, modulation and coding for QoS adaptability, beam-forming, and spacetime coding for MIMO. To maintain link in adverse conditions, wide dynamic range especially for analog-todigital converter (ADC) and high sensitive receiver with rapid adaptation to changes in interference temperature are required.

CR will be also based on strong cross-layer interactions. For example, the cognitive spectrum management involves 


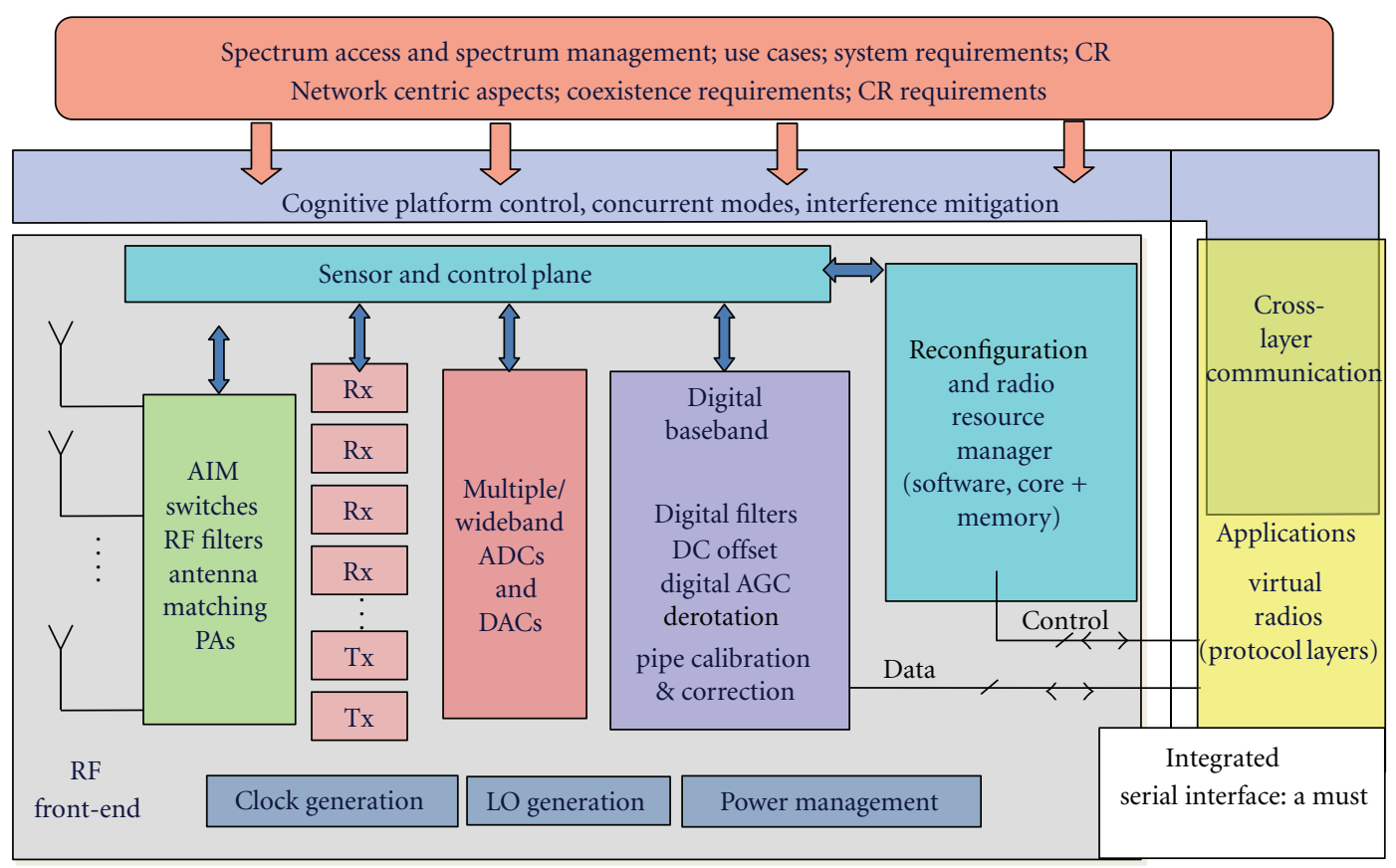

FIGURE 3: Full cognitive radio grail quest.

intelligent use of spectrum based on anticipating the demand for spectrum by the user and previous observation of user behavior. Another cognitive behavior is to monitor the environment in which the CR is operating and then manage the resource intelligently based on expectations or experiences.

5.1. RF Front-Ends Transceiver Challenges. A key bottleneck in CR has always been and continues to be the frequencyagile RF front-ends that can easily be coupled with the parts of the CR that carry out the digital processing - be they pure software systems or a mix of hardware and software. This subsection presents short-/midterm solution/requirements for RF front-end transceiver of CR devices. The state of the art related to this topic is described and design challenges are also presented.

CR transceiver should be able to use any available band, adapt to multiple access methods and adaptive modulation scheme, switch quickly between links, and communicate with 2 or more points at a time. Therefore, the RF section needs to be particularly flexible. In addition, CR receiver should be able to sense unused frequency bands if necessary.

One of the most difficult engineering concerns in the $\mathrm{RF}$ portion of SDR/CR, much more difficult than as in traditional OFDM modems (e.g., in simultaneous communications of diverse RATs), is handling very large peak-toaverage power ratios (PAPRs) [18]. The first issue related to high PAPRs is the efficiency of the PA which suffers dramatically because the PA must be oversized in terms of its average power requirement. In addition to creating design problems for the PA, the high PAPR also requires highly linear upconverters leading to high power consumption.
OFDM modulation and simultaneous usage of multiRATs are also very sensitive to the intermodulation distortion (IMD) that results from mild nonlinearity in the RF. For the RF components in the transmitter, the linearity requirement must be met at reasonably high power levels. With the associated requirement for high efficiency, this places extreme design challenges upon the architectural realization. For the RF components in the receiver, the power levels are much lower but the linearity requirement is even more challenging. This arises from the possible presence of adjacent channel interference from other radio systems, independent but closely located.

Resulting from the possibility of the interfering transmitter location being nearer than the desired transmitter, as well as the possibility of channel shadowing on the desired signal, the adjacent channel blocker is often received with a significantly stronger signal power level than the desired channel. The receiver selectivity becomes therefore more and more important. The linearity requirement for the receiver components must include this anticipated level of blocker power above the desired channel. CR receiver should have a wide dynamic range, that is with the ability to handle a large interferer whilst simultaneously receiving a small wanted signal. It means also that it is able to handle large amplitude signal whilst also having a low noise floor. Two direct consequences of this are difficulty in achieving acceptable noise figure performance for the overall receiver, and difficulty in achieving sufficient dynamic range for the ADCs.

As in OFDM system, phase noise and distortion should be taken carefully in SDR/CR, the problematic is almost the same. In terms of receiver requirements, it should exhibit a good sensitivity, leading to low noise amplifier (LNA) 




FIgURE 4: CR transceiver architectures—wideband radio.

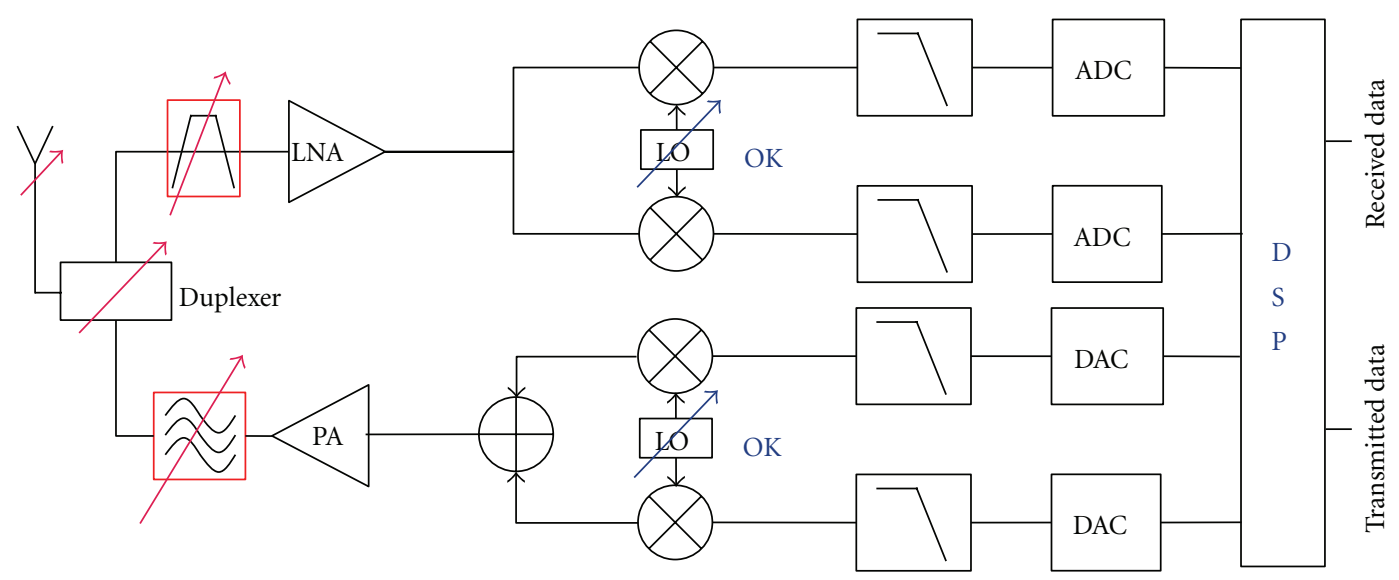

FIGURE 5: CR transceiver architectures-tunable radio.

achieving low noise factor $(\mathrm{NF})(<3 \mathrm{~dB})$ and filter design resulting in low insertion losses $(<1 \mathrm{~dB})$. It needs to achieve a high LNA and mixer linearity (IP3, IP1), adequate filtering, and low local oscillator (LO) phase noise, and spurious to have a good blocker immunity.

In transmitter, the key requirements are high PA linearity as explained above and low PA noise necessary for low adjacent channel leakage power (ACLP), high PA efficiency and heat removal, and low filter insertion loss to reduce power consumption.

Traditional front-end technology cannot handle these requirements because they are generally band limited, both for the form of modulation used and the frequency band in which they operate. Even so called multimode/multiband and wide band transceivers have limitations and generally operate by switching front ends as required [19].

Typically linearity, selectivity, phase distortion, and phase noise issues are addressed through the addition of costly and power-hungry external components including surface acoustic wave (SAW) filters and crystal oscillators that contribute to a higher system bill of materials (BOMs) and increased power consumption and lack of flexibility required for SDR/CR. The combination of innovative architectures and on-chip filters reduce BOM costs and power dissipation; however, the signal corruptions previously discussed can become problematic.

Solutions for improved performance in SoC's perspective include process selection, innovative architectures, the use of on-chip passive components when possible. Although there are some advantages to using a GaAs process for higher linearity or a bipolar process to reduce noise for RF signal processing, the all CMOS radio is the preferred choice for cost, especially when designing radios with significant digital subsections as the case in SDR/CR. Innovative architectures use complex domain $\mathrm{RF}$ and digital signal processing techniques to mitigate the effects of analog imperfections and smart calibration sequences at adequate timeframe to improve performance of the system. In the following, we will present some research directions in all CMOS radio for SDR/CR and associated challenges.

As shown in Figures 4 and 5, CR transceiver architecture can be wideband radio or tunable radio. Considering wideband radio, the challenges are not only on ADC/DAC which is presented in the next subsection, but also the high dynamic range and high linearity LNA, and narrowband impedance matching network. Considering tunable radio, the key issues 




Figure 6: RF transmitter limitations.

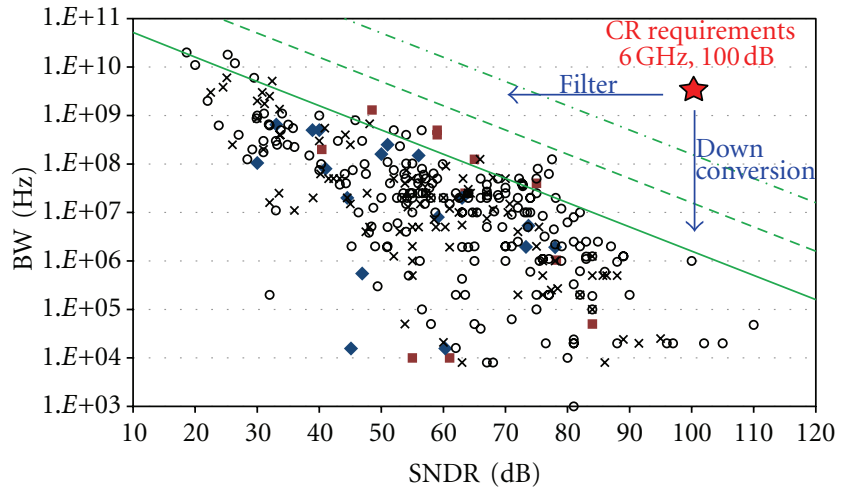

- ISSCC 2011

- VLSI 2011

- Jitter $=1 \mathrm{psrms}$

--- Jitter $=0.1 \mathrm{psrms}$

- ISSCC 1997-2010 - -. Jitter $=0.01$ psrms

$\times$ VLSI 1997-2010

FIGURE 7: ADC SNDR as a function of bandwidth.

are related to the frequency-agile antenna, duplexer, and passive filters. In this direction, some solutions have been proposed, for example, a tunable integrated duplexer in [20], tunable RF BAW filters in [21], using metamaterials as BST in [22], or RF MEMS [23]. However the performances are not good enough and the tuning range is quite small. The challenge is still a low-loss pass-band filter with a small size which has an electrically tunable center frequency with a wide tuning range (of $20 \%$ would be truly disruptive).

An alternative solution for tunable receiver performing blocker rejection is based on circuit architecture using translational loops with feedforward structure [24]. However, it requires very high linearity LNA while sacrificing the NF and introducing an LO leakage issues. The feedback structure proposed in [25] achieves higher out of band (OOB) linearity while maintaining a lower NF. Another solution improving the $\mathrm{OOB}$ linearity and the receiver flexibility has been proposed in [26], using both direct conversion receiver and continuous-time delta sigma converter with upconverted RF feedback loop but for a single band and at the cost of a substantial increase of noise figure due to the feedback loop. The OOB filtering improvement is limited to $15 \mathrm{~dB}$ in practice which limits the OOB linearity enhancement. The future design challenge for $[25,26]$ is related to higher bandwidth operation (up to $40 \mathrm{MHz}$ ). In addition concerning [26], future design challenges are also related to multiband capability, OOB filtering enhancement without compromising the noise figure performances and the power consumption.

Receiver architectures particularly suited to partial SDR make use of impedance reflection from baseband to RF port. Connecting the antenna directly to a passive mixer without an RF LNA provides very increased tuning range and linearity [27]. Complex mixer-first, LNA-less architecture [28], provides very good OOB linearity and low NF with baseband programmable RF band-pass filter, wide tunable frequency range, and complex impedance matching. The design challenge for this approach is related to the extension of frequency range (up to $6 \mathrm{GHz}$ ) without degradation of performances.

The RF transmitter limitations are presented in Figure 6. With the success of the all-digital techniques for RF frequency synthesis in all-digital PLL (ADPLL) [29] and the apparition of digital PA concept showing direct reconstruction of the signal envelope in the RF domain, mostly digital transmitters have been demonstrated such as digital polar transmitter [30] and digital transmitter using direct modulation [31]. Although the design of almost digital transmitter is not as challenging than that of frequency-agile receiver, some issues are still remaining, especially decreased ACLP, increased PA efficiency using digital predistortion technique.

5.2. $A D C$ and DAC Challenges. In ideal SDR and $\mathrm{CR}$, the RF signals are converted into the digital domain as close to the antenna as possible. In this way all the processing is handled by the digital signal processing. In this case, not only must the $\mathrm{ADC}$ and $\mathrm{DAC}$ have $\mathrm{a} 100 \mathrm{~dB}$ dynamic range (with $\mathrm{NF}=$ $4 \mathrm{~dB}$, noise floor in $10 \mathrm{MHz}$ is at $-100 \mathrm{dBm}$, interference and maximum blocker are supposed at $0 \mathrm{dBm}$ ), and be able to operate over a very wide range, extending up to $6 \mathrm{GHz}$ at least, but the transmitter must be able to handle significant levels of power. The power consumption of the ADC could be $1 \mathrm{~kW}$ if the $1 \mathrm{pJ} /$ conversion is assumed.

As illustrated in Figure 7, these requirements are very far beyond the limits of the technology available [32]. Therefore, as mentioned earlier, realistic CR receivers for short-/midterm are based on downconversion and filtering 
TABLE 2: CPU-ARM perforamnces.

\begin{tabular}{|c|c|c|c|c|}
\hline Technology & 2007-8 & $2009-10$ & 2011-12 & Improvement \\
\hline ARM Processor & ARM11 470-700 DMIPS & $\begin{array}{c}\text { Cortex-A8 1,200-2,000 } \\
\text { DMIPS }\end{array}$ & $\begin{array}{c}\text { Dual Cortex-A9 5,000+ } \\
\text { DMIPS }\end{array}$ & $10 \times+\mathrm{SMP}$ \\
\hline Ext. display & VGA & $\mathrm{XGA}$ & WUXGA + HDMI & $8 \times+$ HDMI \\
\hline Video & VGA-30 fps & $720-30 \mathrm{fps}$ & $1080 \mathrm{p}-30 \mathrm{fps}$ & $7 \times$ \\
\hline 3D graphics & 2 Mtri/s OpenGL ES1.1 & 10 + Mtri/s OpenGL ES2.0 & 20+ Mtri/s OpenGL ES2.0 & $10 \times+$ Pgm. shaders \\
\hline Imaging & 3-5 MP & $8-12 \mathrm{MP}$ & $16-20 \mathrm{MP}$ & $7 \times$ \\
\hline Audio & $15 \mathrm{hrs}$ & $40 \mathrm{hrs}$ & $140+$ hrs & $10 \times$ \\
\hline DDR memory & $128-256 \mathrm{MB}$ & $256-512 \mathrm{MB}$ & $1-2 \mathrm{~GB}$ & $8 \times$ \\
\hline Mass storage & $8-16$ GB & $16-32 \mathrm{~GB}$ & $64-128$ GB & $8 \times$ \\
\hline Process & $90 \mathrm{~nm}$ & $65 / 45 \mathrm{~nm}$ & $45 \mathrm{~nm} /$ beyond & $3+$ nodes \\
\hline
\end{tabular}

in front of the $\mathrm{ADC}$ to reduce both the required dynamic range and the conversion bandwidth.

For long-term research, wideband radio with very few amount of off-chip and passive components reduce analog signal processing and is still a desired solution. In this case, the challenge is mainly on ADC/DAC. In this optic, parallelism can be used in order to widen the conversion bandwidth. Parallel continuous-time $\Delta \Sigma$ ADC presented in [33] needs very complex digital synthesis filters which become even more complex when the channel number is increased. Hybrid filter bank based ADC [34] could be a promising solution for CR applications. However it is really sensitive to analog filter errors and imperfections, necessitating high resource calibration technique. The most natural solution employs time-interleaving [35] giving outstanding performance in terms of speed. But it lacks the resolution and dynamic range. The challenges are background calibration of frequency-dependent channel mismatch and time skew errors corrections without the need for any special calibration signal or postproduction trimming. Digital postlinearization is desired to suppress low order nonlinearities of parallel ADCs and nonlinearities caused by preADC analog components. Another technique is to combine time multiplexing and frequency multiplexing by using band-pass charge sampling filters as analysis filters in hybrid filter banks architecture $[36,37]$. This leads to the reduced complexity of analog analysis filters, and at the same time the sensitivity to analog errors and imperfections are reduced. However, a deeper investigation on practical implementation to widen the bandwidth and sensitivity is really needed.

Almost digital RF transmitters employ an RF-DAC [30, 31]. Although some improvements are still encouraged, the design is less challenging than that of ADCs. The key issues in IC design are rather in $\mathrm{Rx}$ and especially with spectrum sensing algorithms implementation: broadband, high linearity, high dynamic range, and low noise.

5.3. Baseband Challenges. Looking at the trend of the digital core processor embedded in expensive smart phone devices, as shown in Table 2, the embedded CPU hosts are more and more powerful. As a result, a part of the digital filters in addition to the demodulation functions are implemented in the software domain. This is already the case in low latency systems as GPS and digital broadcast radio system. This trend is expected to continue paving the way of "true" SDR receiver.

In SDR/CR, the baseband architecture requires dynamic reconfigurations, high computational demand, up to 1 TOPS (100 GOPS are now required for $4 \mathrm{G}$ mobile terminal), and low power (roughly $500 \mathrm{~mW}$ ) under real time constraints. In order to fulfill the unique requirements of embedded applications (real-time, low-power, and multitasking) and improve the flexibility, multiprocessor system-on-chip (MPSoC) has been introduced [38]. network-on-chip (NoC) based architecture can be used to enable fast run-time reconfiguration and reduce the power consumption with distributed power management [39]. 3D NoC is a promising solution for increased modularity and scalability [40]. 3D stacking is a very interesting technology for SoC, however, the matching between $3 \mathrm{D}$ technology and multicore is not straightforward due to some limitations of 3D technology. Dynamic task management has been proposed in [41] to solve MPSoC scalability and programmability problem, and improve resource allocation and energy efficiency.

With real-time constraints, the challenge in baseband architecture and design is to enable flexibility with reduced overhead in terms of performances, powerconsumption, and silicon area by optimizing the tradeoff between performances (dynamic reconfigurations, computational resources) and power efficiency, implementing efficient power management techniques and reducing the run-time management overhead with flexible/dynamic task management.

5.4. Spectrum Sensing Algorithm Implementation. Although, regulatory agencies beginning with FCC and then recently with Ofcom do not require the implementation of spectrum sensing as mandatory but only optional for white space devices operating in the TV bands as a secondary user, spectrum sensing stays a challenging task for CRS and very useful for a better spectrum management and would be a key feature for CR devices in the future. In terms of algorithms, the challenge is on the accuracy on spectrum occupancy decision, sensing time and malicious adversary, taking into account the fundamental limits of spectrum 
TABLE 3: Spectrum sensing—regulation requirements.

\begin{tabular}{lccc}
\hline & FCC 2010 & Ofcom 09 & IEEE 802.22 \\
\hline Max. power adjacent to TV/other $[\mathrm{dBm}]$ & $16 / 17$ & $4 / 17$ & 36 \\
Adjacent-channel emission & $-72 \mathrm{dBc} *$ & $-46 \mathrm{dBc}$ & $-49 \mathrm{dBc}$ \\
Digital TV sensing threshold $[\mathrm{dBm} / \mathrm{MHz}]$ & $-114 / 6$ & $-120 / 8$ & $116 / 6$ \\
Wireless microphone sensing threshold $[\mathrm{dBm} / \mathrm{MHz}]$ & $-107 / 0.2$ & $-126 / 0.2$ & $-10 / 0.2$ \\
Probability detection $/$ probability FalseAlarm & & & $90 \% / 10 \%$ \\
Backoff time(s) & 2 & $<1$ & 2 \\
Sensing frequency (1/minutes) & 1 & 60 & 30 \\
Bandwidth & 6 & 8 & $6,7,8$ \\
Transmit power control & Yes & Yes & Yes \\
Modulation & Free & Free & OFDM \\
\hline
\end{tabular}

${ }^{*}$ Measured in $100 \mathrm{kHz}$ with reference to total power in $6 \mathrm{MHz}$.

sensing algorithms due to noise uncertainty multipath fading and shadowing and hidden PU problem as mentioned above.

A spectrum sensing algorithm is characterized by its detection, false alarm, and miss detection probabilities but also SNR regime, sensing time and frequency, and especially its implementation complexity.

In a practical implementation, it is interesting to build this function with a very low power receiver chain parallel to the main receiver path. Because of the SNR wall issue [15] and particularly the hidden node problem that should be taking and mitigated with additional margin in the threshold detection level, this function requires a very high quality $\mathrm{RF}$ receiver in terms of NF and linearity. Spectrum sensing algorithms are implemented in the baseband. In terms of implementation complexity and power consumption, it depends on the used algorithms, but it is quite simpler compared to conventional baseband signal processing for demodulation. The most challenging task is located in the RF front-end design, in particular low NF, high linearity, and wide dynamic range:

$$
\begin{aligned}
\mathrm{SNR}_{\min }[\mathrm{dB}]= & P_{\min }[\mathrm{dBm}]-\mathrm{NF}[\mathrm{dB}]+174[\mathrm{dBm} / \mathrm{Hz}] \\
& -10 \log _{10} \mathrm{~B}[\mathrm{~dB} \cdot \mathrm{Hz}] .
\end{aligned}
$$

The equation above represents the relation between the minimal SNR value $\left(\mathrm{SNR}_{\min }\right)$ for a given algorithm and the minimal sensing sensitivity or threshold $\left(P_{\min }\right)$, the NF of the receiver (NF) where $B$ is the channel bandwidth to be sensed.

As shown in Table 3 , the spectrum sensing requirements are very stringent with very low sensing threshold. This sensitivity level is even lower in some cases in CEPT-SE43 $(-169 \mathrm{dBm}$ in the worst case). It leads to a very low NF requirement (less than $7 \mathrm{~dB}$ ) and very high out of band linearity. Baseband implementation for algorithm itself must be optimized in terms of computational resources but also power consumption.

\section{Conclusion and Discussion}

This paper presented an overview and challenges of CRS with focus on implementation of cognitive radio. We summarized the status of the related regulation and standardization activity and then pointed out some key research challenges, especially implementation challenges of cognitive radio.

Having three key capabilities such as cognitive capability, reconfigurable capability, and learning capability, CRS has the potential of being a disruptive force within spectrum management. Using SDR for implementing reconfigurable radios, CRS provides additional flexibility and offers improved efficiency to overall spectrum use. It can exploit the spectrum holes or white spaces of licensed spectrum bands provided that it does not cause harmful interference to any primary users in order to significantly improve the efficiency of spectrum usage.

CRS offers also the possibility of flexibly managing the spectrum in a dynamic manner in heterogeneous radio access networks. Through intelligent management mechanisms, frequency bands can be allocated to RATs dynamically in a way such that the capacity of each RAT is maximized and interference is minimized. Network operator may employ different RATs dynamically over time/frequency/location and acquire or exchange the spectrum usage rights. The cognitive devices may autonomously and dynamically adapt to the diverse heterogeneous radio access networks.

CRS covers multidisciplinary areas attracting a large number of researches with many interesting obtained results. The challenges remain numerous, namely, intelligence distribution and implementation, security, delay/protocol overhead, cross-layer design, flexible hardware design, and so forth, CRS will always be limited by physically possible bounds. Limitations depend on the usage model, future standardization and highly on the carrier to noise ratio needed to decode the signal and the signal bandwidth. It is anticipated that semiconductor manufacturers will make steps to improve the selectivity, linearity, and agility performances. But these performances will lead to additional costs that could be offset by new design techniques, and 
for the digital portions, denser CMOS node. The timeframe to make this happen is more linked to the economical and business model for CRS than real technological issues.

\section{Acknowledgments}

The research leading to these results has received funding from the European Community's Seventh Framework Programme (FP7/2007-2013) under Grant agreement SACRA no 249060, from French industry ministry and the ENIAC Joint Undertaking in ARTEMOS Project, and from Institut Telecom in Green RAN ICT-Asia Project.

\section{References}

[1] J. Mitola III and G. Q. Maguire Jr., "Cognitive radio: making software radios more personal," IEEE Personal Communications, vol. 6, no. 4, pp. 13-18, 1999.

[2] S. Haykin, "Cognitive radio: brain-empowered wireless communications," IEEE Journal on Selected Areas in Communications, vol. 23, no. 2, pp. 201-220, 2005.

[3] R. Tandra, S. M. Mishra, and A. Sahai, "What is a spectrum hole and what does it take to recognize one?" Proceedings of the IEEE, vol. 97, no. 5, pp. 824-848, 2009.

[4] M. Mueck, "ETSI TC RRS (Reconfigurable Radio Systems) building standards for SDR and CRS," in Proceedings of the Workshop on Software Defined Radio and Cognitive Radio standardization, Ispra, Italy, 2011.

[5] J. Wang, M. Ghosh, and K. Challapali, "Emerging cognitive radio applications: a survey," IEEE Communications Magazine, vol. 49, no. 3, pp. 74-81, 2011.

[6] "Definitions of Software Defined Radio (SDR) and Cognitive Radio System (CRS)," ITU-R Report SM 2152, 2009.

[7] S. Filin, H. Harada, H. Murakami, and K. Ishizu, "International standardization of cognitive radio systems," IEEE Communications Magazine, vol. 49, no. 3, pp. 82-89, 2011.

[8] C. Moy, "High-level design approach for the specification of cognitive radio equipments management APIs," Journal of Network and Systems Management, vol. 18, no. 1, pp. 64-96, 2010.

[9] "In the matter of unlicensed operation in the TV broadcast bands: second memorandum opinion and order," FCC 10174, Federal Communications Commission, 2010.

[10] Ofcom, "Digital dividend: cognitive access, statement on licence-exempting cognitive devices using interleaved spectrum," 2009.

[11] Ofcom, "Implementing geolocation: Statement," 2011.

[12] "Technical and operational requirements for the possible operation of cognitive radio systems in the white spaces of the frequency band 470-790 MHz," ECC Report 159, 2010.

[13] F. Granelli, P. Pawelczak, R. Venkatesha Prasad et al., "Standardization and research in cognitive and dynamic spectrum access networks: IEEE SCC41 efforts and other activities," IEEE Communications Magazine, vol. 48, no. 1, pp. 71-79, 2010.

[14] M. Mueck, A. Piipponen, K. Kalliojärvi et al., "ETSI reconfigurable radio systems: status and future directions on software defined radio and cognitive radio standards," IEEE Communications Magazine, vol. 48, no. 9, pp. 78-86, 2010.

[15] R. Tandra and A. Sahai, "SNR walls for signal detection," IEEE Journal on Selected Topics in Signal Processing, vol. 2, no. 1, pp. 4-17, 2008.
[16] I. F. Akyildiz, B. F. Lo, and R. Balakrishnan, "Cooperative spectrum sensing in cognitive radio networks: a survey," Physical Communication, vol. 4, no. 1, pp. 40-62, 2011.

[17] P. Pawelczak, K. Nolan, L. Doyle, S. Oh, and D. Cabric, "Cognitive radio: ten years of experimentation and development," IEEE Communications Magazine, vol. 49, no. 3, pp. 90-100, 2011.

[18] S. Zabre, J. Palicot, Y. Louet, C. Moy, and C. Lereau, "Carrier per carrier analysis of SDR signals power ratio," in Proceedings of the SDR Forum Technical Conference, Orlando, Fla, USA, 2006.

[19] T. Sowlati, B. Agarwal, J. Cho et al., "Single-chip multiband WCDMA/HSDPA/HSUPA/EGPRS transceiver with diversity receiver and $3 \mathrm{G}$ digRF interface without SAW filters in transmitter/3G receiver paths," in Proceedings of the IEEE International Solid-State Circuits Conference (ISSCC '09), pp. 116-117, February 2009.

[20] M. Mikhemar, H. Darabi, and A. Abidi, "A tunable integrated duplexer with $50 \mathrm{~dB}$ isolation in 40nm CMOS," in Proceedings of the IEEE International Solid-State Circuits Conference (ISSCC '09), pp. 386-387, February 2009.

[21] S. Razafimandimby, C. Tilhac, A. Cathelin, A. Kaiser, and D. Belot, "Digital tuning of an analog tunable bandpass BAWfilter at GHz frequency," in Proceedings of the 33rd European Solid-State Circuits Conference (ESSCIRC '07), pp. 218-221, September 2007.

[22] K. B. Kim, "Tunable dual-mode filter using varactor and variable ring resonator on integrated BST/TiO2/Si substrate," Electronics Letters, vol. 46, no. 7, pp. 509-511, 2010.

[23] T. Oita, "RF MEMS: focusing on the next step," in Proceedings of the IEEE Ultrasonics Symposium, pp. 1173-1178, 2009.

[24] H. Darabi, "A blocker filtering technique for wireless receivers," in Proceedings of the 54th IEEE International SolidState Circuits Conference (ISSCC '07), pp. 77-588, February 2007.

[25] X. He and H. Kundur, "A compact SAW-less multiband WCDMA/GPS receiver front-end with translational loop for input matching," ISSCC Digest of Technical Papers, pp. 372373, 2011.

[26] K. Koli, S. Kallioinen, J. Jussila, P. Sivonen, and A. Parssinen, "A 900-MHz direct delta-sigma receiver in 65-nm CMOS," IEEE Journal of Solid-State Circuits, vol. 45, no. 12, pp. 2807-2818, 2010.

[27] M. C. M. Soer, E. A. M. Klumperink, Z. Ru, F. E. Van Vliet, and B. Nauta, "A 0.2-to-2.0GHz 65nm CMOS receiver without LNA achieving $>11 \mathrm{dBm}$ IIP3 and $<6.5 \mathrm{~dB}$ NF," in Proceedings of the IEEE International Solid-State Circuits Conference (ISSCC '09), February 2009.

[28] C. Andrews and A. C. Molnar, "A passive mixer-first receiver with digitally controlled and widely tunable RF interface," IEEE Journal of Solid-State Circuits, vol. 45, no. 12, pp. 26962708, 2010.

[29] R. B. Staszewski, "State-of-the-art and future directions of high-performance all-digital frequency synthesis in nanometer CMOS," IEEE Transactions on Circuits and Systems I, vol. 58, no. 7, pp. 1497-1510, 2011.

[30] Z. Boos, A. Menkhoff, F. Kuttner et al., "A fully digital multimode polar transmitter employing $17 \mathrm{~b}$ RF DAC in $3 \mathrm{G}$ mode," in Proceedings of the IEEE International Solid-State Circuits Conference, pp. 376-377, 2011.

[31] A. Pozsgay, T. Zounes, R. Hossain, M. Boulemnakher, V. Knopik, and S. Grange, "A fully digital 65nm CMOS transmitter for the 2.4-to-2.7GHz WiFi/WiMAX bands using $5.4 \mathrm{GHz}$ 
$\triangle \Sigma$ RF DACs," in Proceedings of the IEEE International Solid State Circuits Conference (ISSCC '08), pp. 355-619, February 2008.

[32] B. Murmann, “ADC Performance Survey 1997-2011," http://www.stanford.edu/ murmann/adcsurvey.html.

[33] J. Arias, L. Quintanilla, J. Segundo, L. Enríquez, J. Vicente, and J. M. Hernández-Mangas, "Parallel continuous-time $\Delta \Sigma$ ADC for OFDM UWB receivers," IEEE Transactions on Circuits and Systems I, vol. 56, no. 7, pp. 1478-1487, 2009.

[34] C. Lelandais-Perrault, T. Petrescu, D. Poulton, P. Duhamel, and J. Oksman, "Wideband, bandpass, and versatile hybrid filter bank A/D conversion for software radio," IEEE Transactions on Circuits and Systems I, vol. 56, no. 8, pp. 1772-1782, 2009.

[35] C.-C. Huang, C.-Y. Wang, and J.-T. Wu, "A CMOS 6Bit 16-GS/s time-interleaved ADC using digital background calibration techniques," IEEE Journal of Solid-State Circuits, vol. 46, no. 4, pp. 848-858, 2011.

[36] A. Gruget, M. Roger, V. T. Nguyen, C. Lelandais-Perrault, P. Bénabès, and P. Loumeau, "Wide-band multipath A to D converter for cognitive radio applications," in Proceedings of the IEEE International Microwave Workshop Series on RF Front-Ends for Software Defined and Cognitive Radio Solutions (IMWS '10), pp. 73-76, Aveiro, Portugal, 2010.

[37] A. Gruget, M. Roger, V. T. Nguyen, C. Lelandais-Perrault, P. Bénabès, and P. Loumeau, "Optimization of bandpass charge sampling filters in hybrid filter banks converters for cognitive radio applications," in Proceedings of the 20th European Conference on Circuit Theory and Design (ECCTD '11), pp. 785-788, Linpöping, Sweden, 2011.

[38] W. Wolf, A. A. Jerraya, and G. Martin, "Multiprocessor system-on-chip (MPSoC) technology," IEEE Transactions on Computer-Aided Design of Integrated Circuits and Systems, vol. 27, no. 10, pp. 1701-1713, 2008.

[39] F. Clermidy, C. Bernard, R. Lemaire et al., "A 477mW NoCbased digital baseband for MIMO 4G SDR," in Proceedings of the IEEE International Solid-State Circuits Conference (ISSCC '10), pp. 278-279, February 2010.

[40] F. Clermidy, F. Darve, D. Dutoit, W. Lafi, and P. Vivet, "3D Embedded multi-core: some perspectives," in Proceedings of the Design, Automation and Test in Europe (DATE '11), pp. 1327-1332, 2011.

[41] C. Jalier, D. Lattard, G. Sassatelli, P. Benoit, and L. Torres, "A homogeneous MPSoC with dynamic task mapping for software defined radio," in Proceedings of the IEEE Annual Symposium on VLSI, pp. 345-350, July 2010. 

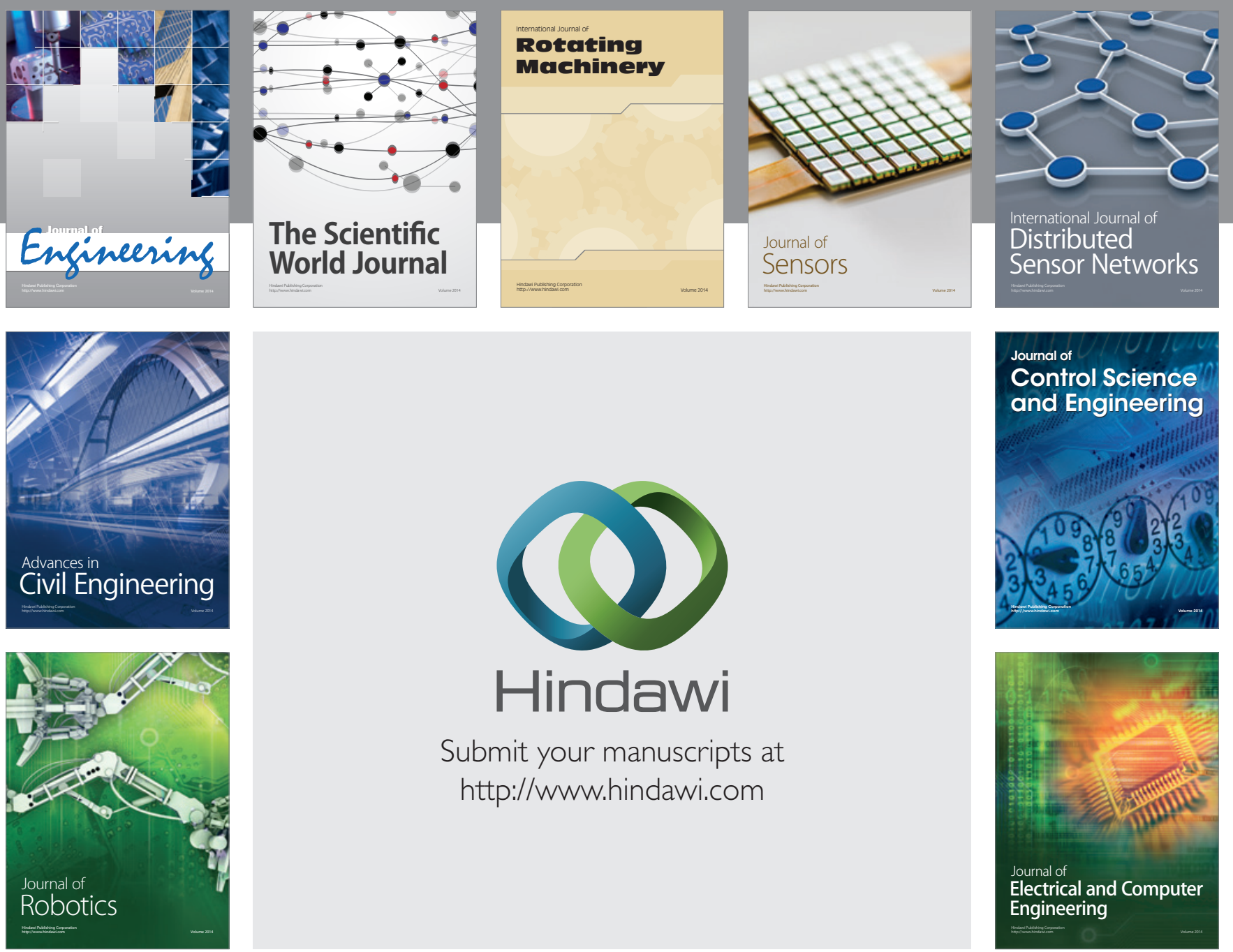

Submit your manuscripts at

http://www.hindawi.com
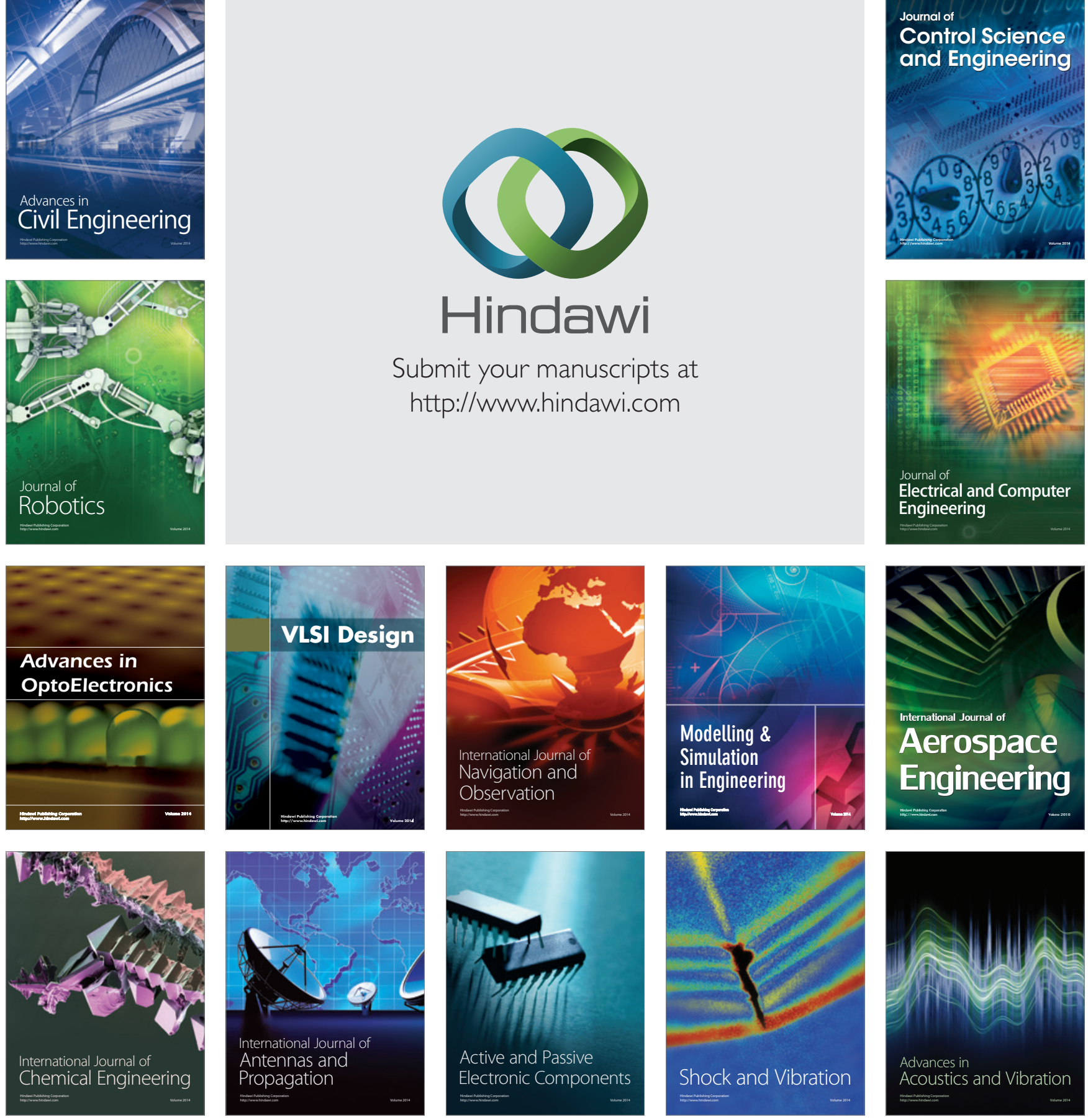